AIAA 2001-2274

\title{
Sound Emission of Rotor Induced Deformations of Generator Casings
}

\author{
W. Polifke * \\ Technische Universität München, \\ D-857/7 Garching, Germany \\ B. Müller ${ }^{\dagger}$ \\ Uppsala University, S-751 04 Uppsala, Sweden \\ H.C. Yee ${ }^{\ddagger}$ \\ NASA Ames Research Center, \\ Moffett Field, CA 94035, USA
}

\begin{abstract}
The casing of large electrical generators can be deformed slightly by the rotor's magnetic fleld. The sound emission produced by these periodic deformations, which could possibly exceed guaranteed noise emission limits, is analysed analytically and numerically. From the deformation of the casing, the normal velocity of the generator's surface is computed. Taking into account the corresponding symmetry, an analytical solution for the acoustic pressure outside the generator is found in terms of the Hankel function of second order. The normal velocity of the generator surface provides the required boundary condition for the acoustic pressure and determines the magnitude of pressure oscillations. For the numerical simulation, the nonlinear $2 \mathrm{D}$ Euler equations are formulated in a perturbation form for low Mach number Computational Aeroacoustics (CAA). The spatial derivatives are discretized by the classical sixth-order central interior scheme and a third-order boundary scheme. Spurious high frequency oscillations are damped by a characteristic-based artificial compression method (ACM) flter. The time derivatives are approximated by the classical 4 th-order Runge-Kutta method. The numerical results are in excellent agreement with the analytical solution.
\end{abstract}

\section{Introduction}

For large air-cooled electrical generators, periodic deformations of the generator casing are induced by the rotor's mannetic field (Fig. 1). Finite element based structural analysis suggests deformation amplitudes relative to the generator radius less than $10^{-5} \mathrm{~m}$, cf. Ahrens ${ }^{1}$. Albeit these deformations are quite small, they are a source of sound. They could possibly exceed guaranteed noise emission limits. In this note we present analytical and numerical solutions of the sound pressure level resulting from rotor induced casing deformations with given amplitude.

\footnotetext{
"Professor, Lehrstuhl für Thermodynamik, polifkeigtd.mw.tum.de

${ }^{\dagger}$ Professor, Department of Scientific Computing, IT, berud@idb.uu.se

\$Senior Research Scientist, Numerical Aerospace Simulation Systems Division, yee@inas.nasa.gov

Copyright (C) 2001 by the American Institute of Aeronantics and Astronautics, Inc. No copyright is asserted in the linited States under Title 17, U.S. Code. The U.S. Government has a royaltyfree licerse to excrcise all rights under the copyright claimed herein for Governmental Purposes. All other rights are reserved by the copyright owner
}

\section{Analytical Solution}

To simplify the problem, we consider a generator of infinite axial extent. The problem then reduces to two dimensions (in the $x, y$ or the $r, \phi$ plane, respectively). The deformation of the casing induced by the rotor's magnetic field provides a (velocity) boundary condition for the acoustic field. Note that only the velocity component normal to the surface of the casing is relevant for the acoustic field. Therefore the acoustic pressure distribution produced by the casing deformations is equivalent to that of a rotating ellipse (Fig. 2).

As a first step we determine the velocity of the surface of the ellipse resulting from its rotation. Without loss of generality, an ellipse oriented as shown in Fig. 2 at time $t=0$ is considered. The points $x_{s}$ on the surface of the ellipse may be described by

$$
\mathbf{x}_{s}=\left(\begin{array}{c}
a \cos \tau \\
b \sin \tau
\end{array}\right)
$$

where $a, b$ are the semi-major and semi-minor axes, respectively, and the parameter $\tau$ runs from 0 to $2 \pi$. We assume that the casing deformations are small in 

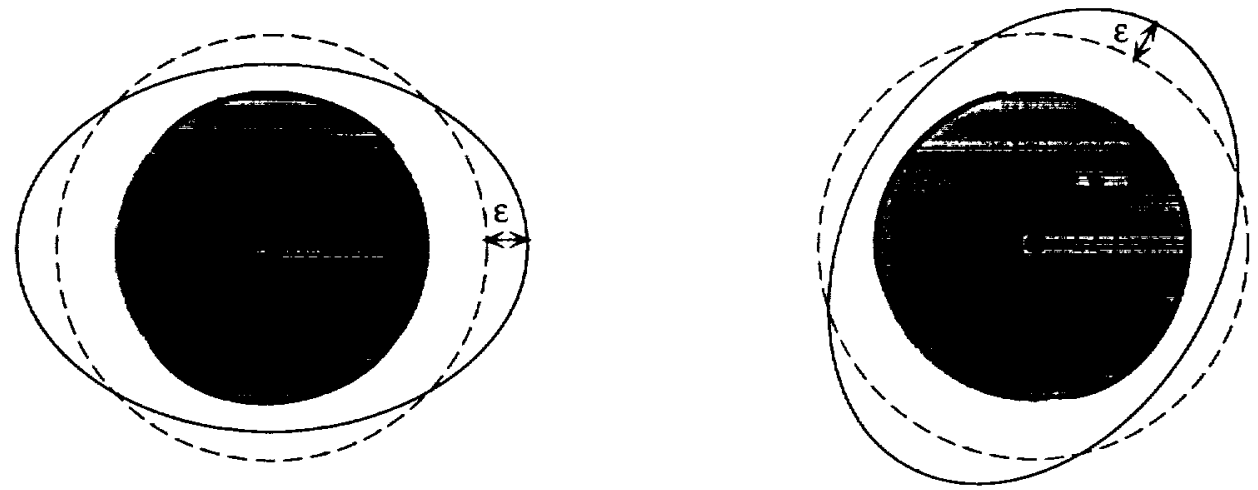

Fig. 1 Schematic of the casing deformation induced by the magnetic field of the rotor. The deformation of the casing follows the rotor's movement.

amplitude, and write

$$
\begin{aligned}
& a=R(1+\epsilon), \\
& b=R(1-\epsilon),
\end{aligned}
$$

with $\epsilon \ll 1$. The following approximations are then accurate to second order in $\epsilon$ :

$$
\begin{aligned}
\tan \phi & \approx(1-2 \epsilon) \tan \tau \\
\tau & \approx \phi+\epsilon \sin 2 \phi
\end{aligned}
$$

where $\phi$ is the azimuth angle, as indicated in Fig. 2.

Using Taylor expansions and trigonometric identities, it is straightforward to show that at time $t=0$ the surface of the ellipse is described by

$$
\mathbf{x}_{s}(\phi, t=0)=R(1+\epsilon \cos 2 \phi)\left(\begin{array}{c}
\cos \phi \\
\sin \phi
\end{array}\right)+\mathcal{O}\left(\epsilon^{2}\right)
$$

At later times $t$, the surface vector $\mathbf{x}_{s}$ at angular position $\phi$ is equal to

$$
\mathbf{x}_{s}(\phi, t)=\mathcal{R}(\Omega t) \mathbf{x}_{s}(\phi-\Omega t, t=0)
$$

where

$$
\mathcal{R}(\theta)=\left(\begin{array}{cc}
\cos \theta & -\sin \theta \\
\sin \theta & \cos \theta
\end{array}\right)
$$

is the transformation matrix that rotates a vector by an angle $\theta$ about the origin in the $(x, y)$ plane. Equation (2) expresses the fact that the ellipse rotates about its center by an angle equal to $\Omega t$ during time $t$. Combining (1) and (2), we obtain

$$
\mathbf{x}_{s}(\phi, t)=R[1+\epsilon \cos 2(\phi-\Omega t)]\left(\begin{array}{c}
\cos \phi \\
\sin \phi
\end{array}\right)+\mathcal{O}\left(\epsilon^{2}\right) \text {. }
$$

Differentiating with respect to time yields the velocity

$$
\mathbf{v}_{s}(\phi, t)=2 \epsilon R \Omega \sin 2(\phi-\Omega t)\left(\begin{array}{c}
\cos \phi \\
\sin \phi
\end{array}\right)+\mathcal{O}\left(\epsilon^{2}\right)
$$

Note that as we consider the movement of points on the ellipse's surface along a given direction (we keep $\phi$ fixed), the velocity vector $v_{s}(\phi, t)$ is aligned with the radial direction. As the ellipse's surface normal $\hat{\mathbf{n}}=$ $(\cos \phi ; \sin \phi)+\mathcal{O}(\epsilon)$ also points approximately in this direction, we obtain for the surface normal velocity

$$
\mathbf{v}_{s} \cdot \hat{\mathbf{n}}(\phi, t) \approx v_{s, r}(\phi, t)=2 \epsilon R \Omega \sin 2(\phi-\Omega t)+\mathcal{O}\left(\epsilon^{2}\right)
$$

for the radial velocity component $v_{s, r}$. Introducing the more convenient complex exponentials instead of the sine function, where it is understood that physical quantities are the real part of complex numbers, we obtain

$$
v_{s, r}(\phi, t)=-i \epsilon R \omega e^{2 i \phi} e^{-i \omega t}+\mathcal{O}\left(\epsilon^{2}\right),
$$

where $\omega \equiv 2 \Omega$ has been introduced. We find that according to (3) and (5), each surface element of the generator oscillates harmonically with amplitude $\epsilon R$ and a frequency $\omega$, which is equal to twice the angular frequency $\Omega$ of the rotor.

Because the displacement of the generator surface is small, it is appropriate to solve for the distribution of pressure disturbances outside a circular disk

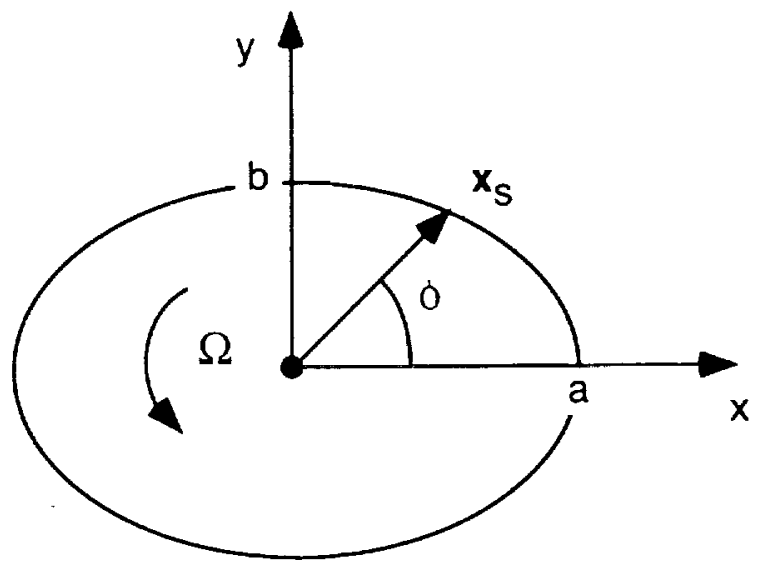

Fig. 2 Schematic and relevant notation of the equivalent problem of a rotating ellipse. 
of radius $R$ and enforce the boundary condition (5) at $r=R$. The acoustic fluctuations of pressure $p^{\prime}$ obey the Helmholtz equation, which reduces for the harmonic case under consideration to

$$
\nabla^{2} p^{\prime}+k^{2} p^{\prime}=0
$$

with the wave number $k=\omega / c$ ( $c$ is the speed of sound). After separation of variables

$$
p^{\prime}(r, \phi)=\varrho(r) \Phi(\phi)
$$

one obtains

$$
\frac{1}{r} \frac{\partial}{\partial r}\left(r \frac{\partial \varrho}{\partial r}\right)+\left(k^{2}-\frac{m^{2}}{r^{2}}\right) \varrho=0 .
$$

Due to the angular dependence given by (5), $\Phi(\phi)=$ $\exp \{2 i \phi\}$ and $m=2$. The general solution for the radial function $\varrho(k r)$ is then (see Morse \& Ingard, Sect. $\left.7.3^{2}\right)$

$$
\varrho(k r)=A H_{2}^{(1)}(k r) .
$$

where $H_{m}^{(1)}=J_{m}+i N_{m}$ is the Hankel function of $m$ th order $\left(J_{m}\right.$ and $N_{r n}$ are Bessel and Neumann functions, respectively), and $A$ is a constant which has to be determined from boundary conditions. At the (impermeable) surface of the generator,

$$
v_{s, r}=u_{r}^{\prime},
$$

and the linearized form of the radial momentum equation

$$
\rho \frac{\partial u_{r}^{\prime}}{\partial t}=-\frac{\partial p^{\prime}}{\partial r}
$$

yields then with (5) the relations required to fix the constant $A$. We obtain the simple result

$$
A=\frac{\rho \epsilon \omega^{2} R}{k H_{2}^{(1)}(k R)}
$$

where ", denotes the derivative of a function with respect to its argument.

The problem is non-dimensionalized conveniently with the generator radius $R$, the speed of sound $c$ and the density $\rho$. The pressure fluctuations $p^{\prime}$ are nondimensionalized with $\rho c^{2}$. Introducing the Helmholtz number $\mathcal{H} \equiv k R$, we obtain finally

$$
\bar{p}^{\prime}(\bar{r}, \phi)=\frac{\epsilon \mathcal{H}}{H_{2}^{(1)}(\mathcal{H})} H_{2}^{(1)}(\mathcal{H} \tilde{r}) \exp \{2 i \phi\} .
$$

where the tilde indicates non-dimensionalized quantities. Numerical values for the derivatives $H_{2}^{(1) \prime}(z)=$ $i E_{2}(z) \exp \left\{i \gamma_{2}(z)\right\}$ of the Hankel functions are given in Table $V$ of Morse and Ingard ${ }^{2}$ or can be computed with standard mathematical software.

It has been shown by Mïller $^{3}$ that the normal velocity of the surface of an almost circular Kirchhoff vortex is also described by (5). Hence the acoustic pressure distribution (10) produced by a rotating ellipse of small eccentricity is identical to that of a Kirchhoff vortex of equal size, eccentricity and rotational speed. Farfield and nearfield approximations of the pressure distribution (10) are also presented in Müller $^{3}$, and it is pointed out that a farfield approximation derived using Green's function yields identical results.

However, for large electrical generators used in power generation, typical Helmholtz numbers $\mathcal{H}$ might range from 1 to 5 . With $\mathcal{H} \sim \mathcal{O}(1)$, "small-source" or "far-field" approximations are not applicable when the pressure distribution in the vicinity of the generator has to be determined.

\section{Numerical Solution}

We follow the approach outlined by Müller and $\mathrm{Yee}^{16}$ for the Kirchhoff vortex sound. Before the application of the numerical scheme, we conditioned the nonlinear $2 \mathrm{D}$ Euler equations by reformulating the equations in a perturbation form for low Mach number Computational Aeroacoustics(CAA), cf. Sesterhenn et al. ${ }^{4}$. The unknowns are the changes of the conservative variables with respect to their stagnation values. This formulation retains all nonlinearities and the conservation form, while numerical cancellation errors are minimized compared to the original conservative laws. The Euler equations in this perturbation form can be written as

$$
\begin{aligned}
\frac{\partial \rho^{\prime}}{\partial t}+\nabla \cdot(\rho \mathbf{u})^{\prime} & =0 \\
\frac{\partial(\rho \mathbf{u})^{\prime}}{\partial t}+\nabla \cdot(\rho \mathbf{u})^{\prime} \mathbf{u}^{\prime}+\nabla p^{\prime} & =0 \\
\frac{\partial(\rho E)^{\prime}}{\partial t}+\nabla \cdot\left((\rho H)^{\prime} \mathbf{u}^{\prime}+(\rho H)_{0} \mathbf{u}^{\prime}\right) & =0
\end{aligned}
$$

where

$$
\begin{aligned}
\rho^{\prime}=\rho-\rho_{0}, & (\rho \mathbf{u})^{\prime}=\rho \mathbf{u}, \quad(\rho E)^{\prime}=\rho E-(\rho E)_{0}, \\
\mathbf{u}^{\prime}=\frac{(\rho \mathbf{u})^{\prime}}{\rho_{0}+\rho^{\prime}}, & p^{\prime}=(\gamma-1)\left[(\rho E)^{\prime}-\frac{1}{2}(\rho \mathbf{u})^{\prime} \cdot \mathbf{u}^{\prime}\right], \\
(\rho H)^{\prime} & =(\rho E)^{\prime}+p^{\prime} .
\end{aligned}
$$

Here, $\rho$ denotes the density, $u$ the velocity, $E$ the total energy per unit mass, $H$ the total enthalpy, and $\gamma=1.4$ the ratio of specific heats for air at standard conditions. The " $"$ " and subscript " 0 " denote perturbation and stagnation variables, respectively.

Although this perturbed form is identical to the original conservation laws, discretizing e.g. $\nabla p$ leads to cancellation errors, whereas these errors are avoided when discretizing $\nabla p^{\prime}$. In Cartesian coordinates, the perturbed 2D Euler equations can be expressed as

$$
\frac{\partial \mathbf{U}^{\prime}}{\partial t}+\frac{\partial \mathbf{F}_{1}^{\prime}}{\partial x}+\frac{\partial \mathbf{F}_{2}^{\prime}}{\partial y}=0
$$


where

$$
\begin{gathered}
\mathbf{U}^{\prime}=\left(\begin{array}{l}
\rho^{\prime} \\
(\rho u)^{\prime} \\
(\rho v)^{\prime} \\
(\rho E)^{\prime}
\end{array}\right), \mathbf{F}_{1}^{\prime}=\left(\begin{array}{l}
(\rho u)^{\prime} \\
(\rho u)^{\prime} u^{\prime}+p^{\prime} \\
(\rho v)^{\prime} u^{\prime} \\
(\rho H)^{\prime} u^{\prime}+(\rho H)_{0} u^{\prime}
\end{array}\right) \\
\mathbf{F}_{2}^{\prime}=\left(\begin{array}{l}
(\rho v)^{\prime} \\
(\rho u)^{\prime} v^{\prime} \\
(\rho v)^{\prime} v^{\prime}+p^{\prime} \\
(\rho H)^{\prime} v^{\prime}+(\rho H)_{0} v^{\prime}
\end{array}\right)
\end{gathered}
$$

Here, $u^{\prime}=u$ is the $x$-direction velocity and $v^{\prime}=v$ is the $y$-direction velocity.

For the treatment of general geometries, a coordinate transformation $(x(\xi, \eta), y(\xi, \eta))$ is used. The resulting transformed $2 \mathrm{D}$ Euler equations, transformed variables and fluxes are

$$
\begin{gathered}
\frac{\partial \hat{\mathbf{U}}^{\prime}}{\partial t}+\frac{\partial \hat{\mathbf{F}}_{1}^{\prime}}{\partial \xi}+\frac{\partial \hat{\mathbf{F}}_{2}^{\prime}}{\partial \eta}=0, \\
\hat{\mathbf{U}}^{\prime}=J^{-1} \mathbf{U}^{\prime}, \\
\hat{\mathbf{F}}_{1}^{\prime}=J^{-1} \xi_{x} \mathbf{F}_{1}^{\prime}+J^{-1} \xi_{y} \mathbf{F}_{2}^{\prime}, \\
\hat{\mathbf{F}}_{2}^{\prime}=J^{-1} \eta_{x} \mathbf{F}_{1}^{\prime}+J^{-1} \eta_{y} \mathbf{F}_{2}^{\prime},
\end{gathered}
$$

with the Jacobian determinant of the transformation $J^{-1}=x_{\xi} y_{\eta}-x_{\eta} y_{\xi}$, and the metric terms $J^{-1} \xi_{x}=y_{\eta}$, $J^{-1} \xi_{y}=-x_{\eta}, J^{-1} \eta_{x}=-y_{\xi}, J^{-1} \eta_{y}=x_{\xi}$.

Kreiss and Scherer ${ }^{5}$, Strand ${ }^{6}$ and Carpenter et al. ${ }^{7}$ constructed high order difference operators $Q$ for " $d / d x$ " such that the summation-by-parts (SBP) principle holds, i.e.

$$
(u, Q v)_{h}=u_{N} v_{N}-u_{0} v_{0}-(Q u, v)_{h},
$$

where $u, v \in \mathrm{R}^{\mathrm{v}+1}, h$ is the uniform grid spacing and $N+1$ is the number of grid points. The discrete scalar product and norm are defined by

$$
(u, v)_{h}=h u^{T} H u, \quad\|u\|_{h}^{2}=(u, u)_{h},
$$

where $H$ is a symmetric positive definite $(N+1) \times$ $(N+1)$ matrix.

We employ a $Q$ operator, which is third-order accurate near the boundary and compatible with the standard sixth-order central difference operator in the interior. It was derived by $\operatorname{Strand}^{6}$ and $Q_{x}$, the $x$ direction $Q$ operator for $\frac{\partial}{\partial x}$ is of the form

$\left(Q_{x} v\right)_{j}= \begin{cases}\frac{1}{h} \sum_{k=0}^{3} d_{j k} v_{k}, & j=0, \ldots, 5, \\ \left(Q_{x}^{(6)} v\right)_{j}, & j=6, \ldots, N-6, \\ -\frac{1}{h} \sum_{k=0}^{8} d_{N-j . k} v_{N-k}, & j=N-5, \ldots, N,\end{cases}$

where $\left(Q_{x}^{(6)} v\right)_{j}=\frac{1}{h}\left(\frac{1}{60} v_{j+3}-\frac{3}{20} v_{j+2}+\frac{3}{4} v_{j+1}-\frac{3}{4} v_{j-1}+\right.$ $\left.\frac{3}{20} v_{j-2}-\frac{1}{60} v_{j-3}\right)$ is the standard sixth-order central difference approximation of the first derivative. The forms of the $5 \times 9$ matrix $D=\left(d_{j k}\right)$ and matrix $H$ can be found in Strand ${ }^{6}$ and Gerritsen and Olsson ${ }^{8}$. Here $H$ is a diagonal matrix defining the norm of the $Q$ operator. The global order of accuracy for (17) is four. Since (17) is based on a diagonal norm, its application to multi-dimensions is straightforward. The metric terms are discretized by the same difference operators as the flux derivatives in (15). In order to guarantee freestream preservation in $3 \mathrm{D}$ for high order spatial discretizations, the metric term treatment of Vinokur and Yee ${ }^{11}$ is recommended.

With the density, velocity and pressure nondimensionalized with reference quantities $\rho_{0}, c_{0}$ and $\rho_{0} c_{1)}^{2}$, respectively, the Riemann invariants can be expressed as $p^{\prime}-u_{n}, p^{\prime}-\rho^{\prime}, u_{t}$ and $p^{\prime}+u_{n}$. Here, $u_{n}$ is the normal velocity and $u_{t}$ is the tangential velocity. At the generator, the in-going Riemann invariants are prescribed using the analytical solution (10), cf. Müller and $\mathrm{Yee}^{16}$. At the farfield, nonreflecting boundary conditions are implemented by setting the in-going Riemann invariants equal to zero corresponding to stagnation conditions. The outgoing Riemann invariants are taken from the numerical solution computed at the boundary. We use the so-called injection method to prescribe the in-going Riemann invariants, i.e. by imposing them explicitly which might destroy strict stability. Research is in progress to implement the in-going Riemann invariants by the penalty method called "simultaneous approximation term" (SAT) of Carpenter et al. $^{7}$ or the projection method of Olsson ${ }^{12,13}$. Either approach yields strict stability for a $Q$ operator satisfying the SBP principle (16) for linear problems. For nonlinear systems of conservation laws, the in-going Riemann invariants have to be implemented by the SAT or projection method via a split form of the inviscid flux derivatives ${ }^{14,15}$ (entropy splitting $\left.{ }^{10}\right)$. This will be presented in Miiller and $\mathrm{Yee}^{20}$

The application of the spatial discretization of the perturbed Euler equations in transformed coordinates (15) results in a semi-discrete system of nonlinear ODEs

$$
\frac{d \mathrm{U}}{d t}=\mathbf{R}(\mathrm{U})
$$

where $U$ is the vector of the difference approximations $U_{j, k}^{\prime}$ and $R$ is the vector of two-dimensional spatial difference operators operating on $\frac{\partial \hat{\mathbf{F}}_{1}^{\prime}}{\partial \xi}$ and $\frac{\partial \hat{\mathbf{F}}_{2}^{\prime}}{\partial \eta}$ with each element $\mathbf{R}_{j, k}=-J_{j, k}\left[Q_{\xi} \hat{\mathbf{F}}_{1}^{\prime}+Q_{\eta}^{(6)} \hat{\mathbf{F}}_{2}^{\prime}\right]_{j, k}$. The periodic boundary conditions in the circumferential $\eta$-direction in our application allow the use of the standard sixth-order difference operator $Q_{\eta}^{(6)}$. The ODE system (18) is solved by the 4th-order classical Runge-Kutta method with a CFL condition of 1.783 . To minimize the loss of the global order of accuracy, we impose the time-dependent physical boundary conditions (the in-going Riemann invariants in our case) only after the completion of the full step of the Runge- 
Kutta method. The $Q$ boundary difference operator is, however, employed at every stage of the Runge-Kutta method. Other remedies are discussed in ${ }^{17} 19$.

For long time wave propagation of nonlinear systems, even with the absence of shock waves and/or steep gradients, spurious high frequency oscillations are generated by non-dissipative central spatial schemes. To suppress these spurious oscillations, a modified version of the Yee et al. ${ }^{9} 11$ high order artificial compressibility method (ACM) filter scheme is used. The original AC.I filter scheme involves high order non-dissipative spatial base schemes and a c4aracteristic-based ACM filter. The ACM filter consists of a product of numerical dissipations and an ACM sensor. The ACM sensor consists of a product of a sensor coefficient and a gradient-like detector. Here, the limiter of the numerical dissipation, and the Roe's averaged states are not used. For low Mach number CAA, the limiter which is designed for capturing discontinuities might not be necessary, unless shear layers, high gradient regions and/or boundary layers are present.

At the completion of a full step of the Runge-Kutta method, the numerical solution $\mathbf{U}_{j, k}^{\prime n+1}$ is filtered by

$$
\tilde{\mathbf{U}}_{j, k}^{\prime n+1}=\mathbf{U}_{j, k}^{\prime n+1}-\Delta t J_{j, k}\left[D_{\xi} \mathbf{U}^{\prime}+D_{\eta} \mathbf{U}^{\prime}\right]_{j, k}^{n+1} \text {. }
$$

$D_{\xi} \mathrm{U}_{j, k}^{\prime}$ represents the fourth-order numerical dissipation in the $\xi$-direction defined by

$$
D_{\xi} \mathbf{U}_{j, k}^{\prime}=\kappa \delta_{\xi} \mathbf{R}_{\xi} \mathbf{C}_{\xi}\left|\boldsymbol{\Lambda}_{\xi}\right| \delta_{\xi}^{2} \mathbf{R}_{\xi}^{-1} \delta_{\xi} \mathbf{U}_{j, k}^{\prime}
$$

where $\delta_{\xi}$ is the difference operator given by $\delta_{\xi} \mathbf{U}_{j, k}^{\prime}=$ $\mathbf{U}_{j+1 / 2, k}^{\prime}-\mathbf{U}_{j-1 / 2, k}^{\prime}$. Applying $\delta_{\xi}$ four times yields the standard central difference approximation $\delta_{\xi}^{4} \mathbf{U}_{j, k}^{\prime}=$ $\mathbf{U}_{j+2, k}^{\prime}-4 \mathbf{U}_{j+1, k}^{\prime}+6 \mathbf{U}_{j, k}^{\prime}-4 \mathbf{U}_{j-1, k}^{\prime}+\mathbf{U}_{j-2, k}^{\prime}$ of $\Delta \xi^{+} \frac{\sigma^{4} \mathrm{U}^{\prime}}{\partial \xi^{\top}}(x(j \Delta \xi), y(k \Delta \eta), t)$. Here $D_{\xi} \mathrm{U}^{\prime}{ }_{j, k}$ involves differences of average states. The columns of $\mathbf{R}_{\xi}$ are the right eigenvectors of the Jacobian matrix $\frac{\partial \hat{F}_{1}}{\partial U}$ and may be found in Vinokur and Yee ${ }^{11}$. The eigenval ues of $\frac{\partial \hat{\mathbf{F}}_{1}}{\partial \mathbf{U}}$ define the diagonal matrix $\Lambda_{\xi}=\operatorname{diag}\left(u_{\xi}-\right.$ $\left.c_{\xi}, u_{\xi}, u_{\xi}, u_{\xi}+c_{\xi}\right)$, where $u_{\xi}=u J^{-1} \xi_{x}+v J^{-1} \xi_{y}$, and $c_{\xi}=c \sqrt{\left(J^{-1} \xi_{x}\right)^{2}+\left(J^{-1} \xi_{y}\right)^{2}}$. Instead of the Roeaverage, the $R_{\xi}$ and $\Lambda_{\xi}$ are evaluated at the arithmetic average. Here the ACM sensor is the product of a sensor coefficient $\kappa$ and a gradient-like detector. A simplified form of the the gradient-like detector is expressed by the diagonal matrix $\mathbf{C}_{\xi}$. The $l$ th diagonal element $c^{I}$ of $\mathrm{C}_{\xi}$ is defined by

$$
c_{j+1 / 2, k}^{l}=\max \left(\hat{\theta}_{j, k}, \hat{\theta}_{j+1, k}\right)
$$

where $\hat{\theta}_{j, k}=\frac{|| \alpha_{j+1 / 2, k}^{\prime}|-| \alpha_{j-1 / 2, k}^{\prime}||}{\max \left(\left|\alpha_{j+1 / 2, k}^{1}\right|+\left|\alpha_{j-1 / 2, k}^{\prime}\right| 10^{-7}\right)}$. To avoid an extra logical statement, the quantity $10^{-7}$ is used to avoid division by zero. $\alpha_{j+1 / 2, k}^{I}$ is the wave strength of the $l$ th wave, i.e. the $\mathrm{th}_{\mathrm{th}}$ component of
$\mathbf{R}_{\xi}^{-1}{ }_{j+1 / 2, k} \delta_{\xi} \mathbf{U}_{j, k}^{\prime} . \quad D_{\eta} \mathbf{U}_{j, k}^{\prime}$ is defined analogously. For our numerical experiments, selected nonzero positive ACM sensor coefficient $\kappa$ less than or equal to 0.10 are evaluated.

Although Müller and Yee ${ }^{20}$ performed numerical studies on a similar almost circular Kirchhoff vortex model, aside from application specific, the present simulation differs from Muller and Yee in two ways. The fourth-order classical Runge-Kutta temporal discretization and the full ACM sensor ( $\kappa$ and $C_{\xi}$ ) are evaluated.

\section{Numerical Results}

We consider an elliptic generator casing with radius $R=2 \mathrm{~m}, \epsilon=0.00125, \Omega=82.5 \frac{1}{\mathrm{~s}}$. The stagnation conditions are $\rho_{0}=1.3 \frac{\mathrm{kg}}{\mathrm{m}^{3}}, c_{0}=330 \frac{\mathrm{m}}{\mathrm{s}}$. Thus, the Helmholtz number becomes $\mathcal{H}=k R=2 \Omega R / c_{0}=1$. The time step is chosen as $\Delta t=0.15$. A polar grid mildly stretched near the generator casing in the radial direction, and uniform in the circumferential direction of $129 \times 24$ is used. As presented later, a grid refinement study indicates that this grid is sufficient to capture the flow physics accurately.

We consider the elliptic generator casing started instantaneously. The initial conditions are $\rho^{\prime}=u^{\prime}=$ $v^{\prime}=p^{\prime}=0$, except for the circle $r=R$, where the analytical solution is prescribed. At $t=30$, the generator has rotated 7.5 radians. Figures 3 and 4 show the numerical results without and with characteristicbased filter, respectively. Without filter, the numerical solution is polluted by spurious oscillations. These are eliminated by the filter. The wave front started at $r=R$ at $t=0$ has reached $r \approx 16$ at $t=30$. The numerical solution with filter is compared with the analytical solution along the positive $x$-axis at $t=30$ in Fig. 5. Between $x=1$ and $x \approx 14$, the analytical and numerical solutions are in close agreement. At $x \approx 16$, we see that in general the wavefront cannot match the infinitely long rotating generator solution, because the instantaneously started generator has zero acoustic pressure downstream of the wavefront. The discrepancies, therefore, have physical reasons.

At $t=75$, the generator has rotated 18.75 radians, Now, the wavefront is at $r \approx 38.5$. If the wavefront were reflected at the farfield $r=32.1875$, we should see it at $r \approx 25.875$. The computed acoustic pressure along the positive $x$-axis shown in Fig. 6 is in excellent agreement with the analytical solution, if the characteristic-based ACM filter with a uniform sensor coefficient $\kappa=0.05$ is used. Without the filter, spurious oscillations occur. The wavefront generated at $t=0$ by the elliptic generator casing has passed through the farfield without visible reflection. The quadrupole structure of the acoustic pressure is correctly recovered by the high order $Q$ operator with the characteristic-based ACM filter of $\kappa=0.05$. See Figs. 7 and 8 for the comparison. 


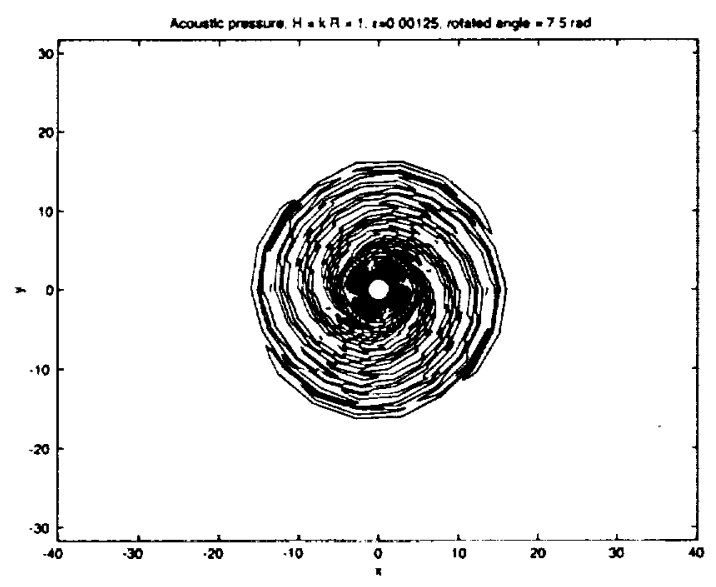

Fig. 3 Computed acoustic pressure contours without fiter for instantaneously started generator with $\mathcal{H}=1, \epsilon=0.00125$, rotated angle $=7.5 \mathrm{rad}$.

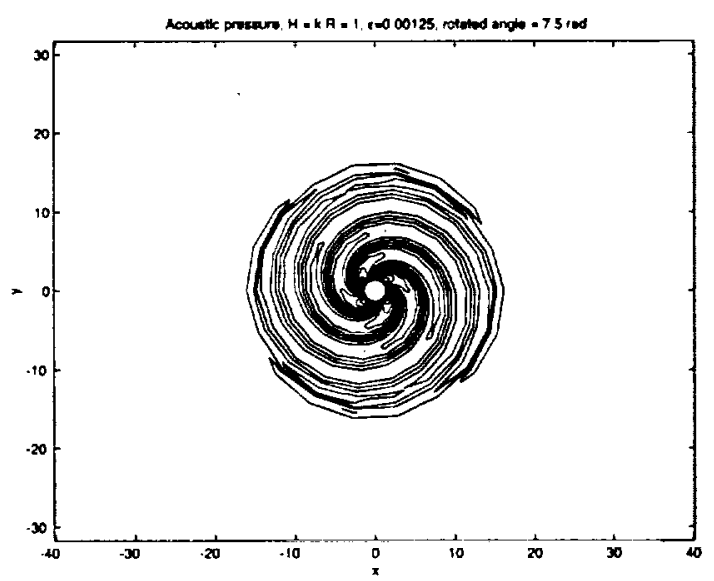

Fig. 4 Computed acoustic pressure contours with filter $(\kappa=0.05)$ for instantaneously started generator with $\mathcal{H}=1, \epsilon=0.00125$, rotated angle $=7.5 \mathrm{rad}$.

We note that the multistage method used in Müller and $\mathrm{Yee}^{16}$ for time integration yields almost identical results as the classical Runge-Kutta method employed here. Since both ODE solvers coincide for linear problems and we are solving essentially a linear problem, it is not surprising that the results with the two different time integrations differ 3 to 4 orders of magnitude less from each other than from the analytical solution.

A grid refinement study was performed with $65 \times 15$, $129 \times 24$ and $257 \times 42$ grids keeping the CFL number constant. The maximum CFL numbers in the radial and circumferential directions were about 1 and 0.2 , respectively. The high order $Q$ operator with the characteristic-based ACM filter of $\kappa=0.05$ was used. The computed acoustic pressure along the positive $x$-axis after time $t=75$ in Fig. 9 indicates grid convergence. The $l_{2}$-norm of the error of $p^{\prime}$ along the positive $x$-axis, i.e. the deviation of the computed from

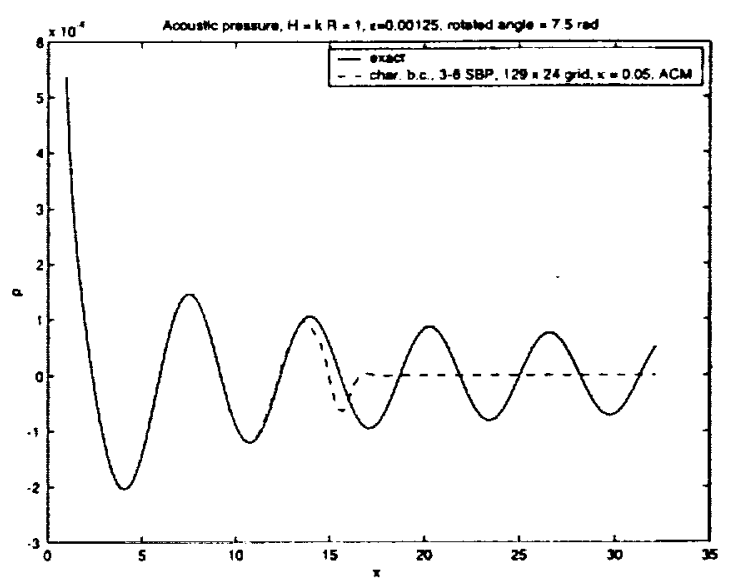

Fig. 5 Comparison of acoustic pressure for instantaneously started generator with $\mathcal{K}=1, \epsilon=0.00125$, rotated angle $=7.5 \mathrm{rad}$.

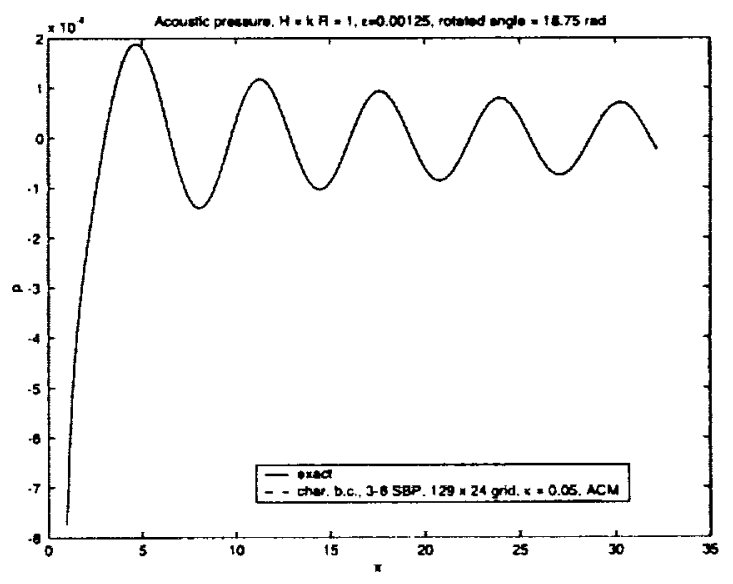

Fig. 6 Comparison of acoustic pressure for instantaneously started generator with $\mathcal{H}=1, \epsilon=0.00125$, rotated angle $=18.75 \mathrm{rad}$.

the analytical acoustic pressure there, has a convergence rate of 4.7 from the course to the medium grid and of 1.9 from the medium to the fine grid. The error of $p^{\prime}$ along the positive $x$-axis is shown in Fig. 10. The reduction in convergence rate seems to be due to the boundary treatment, because the error is largest near the boundaries. A similar drop of convergence rate is observed for the conservative variables in the whole domain as well.

The infuence of the gradient-like detector of the ACM sensor and its sensor coefficient $\kappa$ is investigated. We consider the $129 \times 24$ grid for the same test case as above. This test case consists of smooth flow and it is not a very long time wave propagation simulation, consequently, only a small sensor coefficient is needed. With a small sensor coefficient, the role of the gradient detector is diminishing. This is due to the fact that the gradient-like detector is designed to minimize 


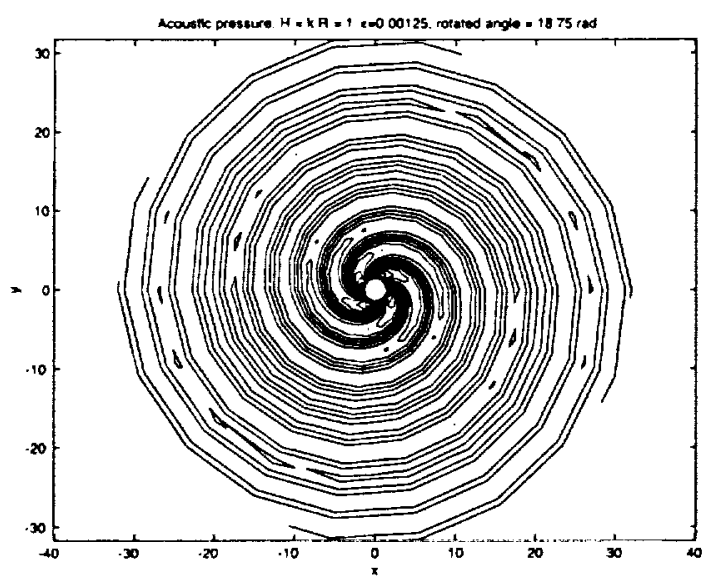

Fig. 7 Acoustic pressure contours with filter $(\kappa=0.05)$ for instantaneously started generator with $\mathcal{H}=1, \epsilon=0.00125$, rotated angle $=18.75 \mathrm{rad}$.

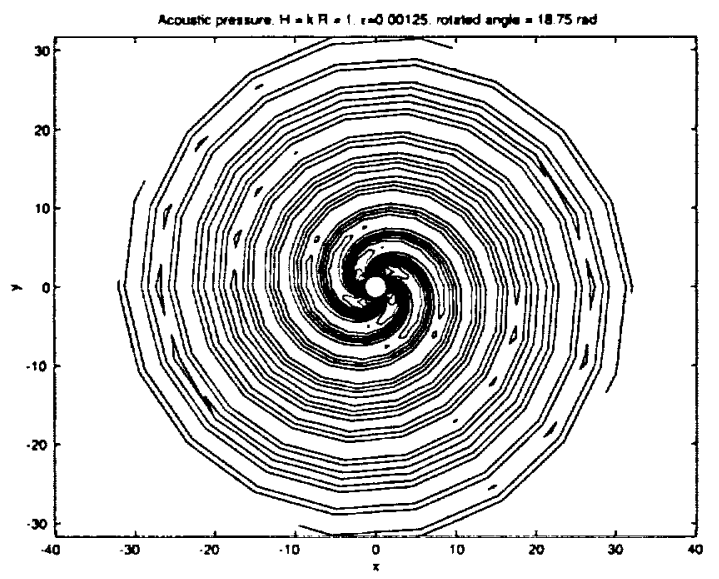

Fig. 8 Exact acoustic pressure contours for generator with $\mathcal{H}=1, \epsilon=0.00125$, rotated angle $=18.75 \mathrm{rad}$.

numerical dissipation away from shocks, shears, high gradient and steep boundary-layer flows with the use of a larger sensor coefficient. However, for very long time wave propagation problems and/or with unknown solution behavior, the full ACM sensor or wavelet sensor (cf. Sjögreen and $\mathrm{Yee}^{21}$ ) is necessary, even for low Mach number CAA (cf. Yee et al. ${ }^{10}$ ). As expected, the difference in results with and without the gradient-like detector $\left(\mathbf{C}_{\xi}\right.$ of $(20)$ vs. $\mathbf{C}_{\xi}=I$ ) are not pronounced for the very small sensor coefficients studied. For $\kappa=0.025$, the error of the acoustic pressure is rougher with the gradient detector than without, cf. Fig. 11. For $\kappa=0.05$, the error of the acoustic pressure with the gradient detector and without behave similarly. However, for $\kappa=0.1$, the error of the acoustic pressure is larger without the gradient detector than with the detector, cf. Fig. 12. In this case, the gradient-like detector is performing its duty

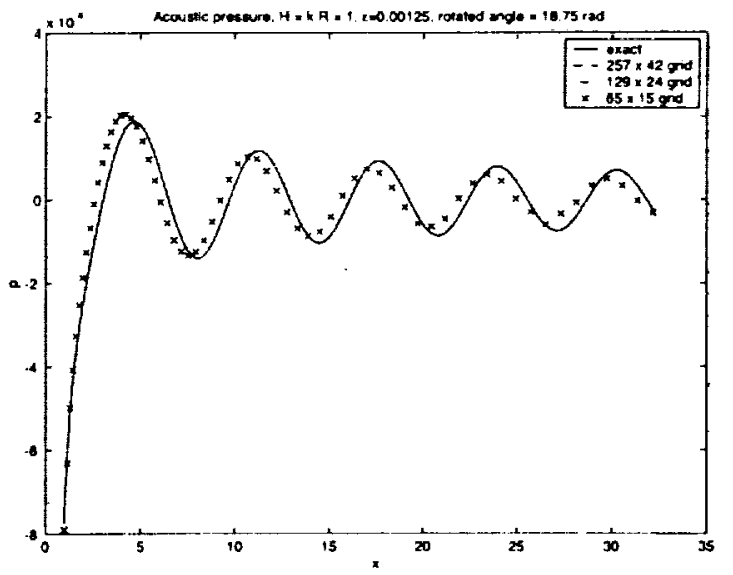

Fig. 9 Grid refinement study of acoustic pressure for instantaneously started generator with $\mathcal{H}=1$, $\epsilon=0.00125$, rotated angle $=18.75 \mathrm{rad}$.

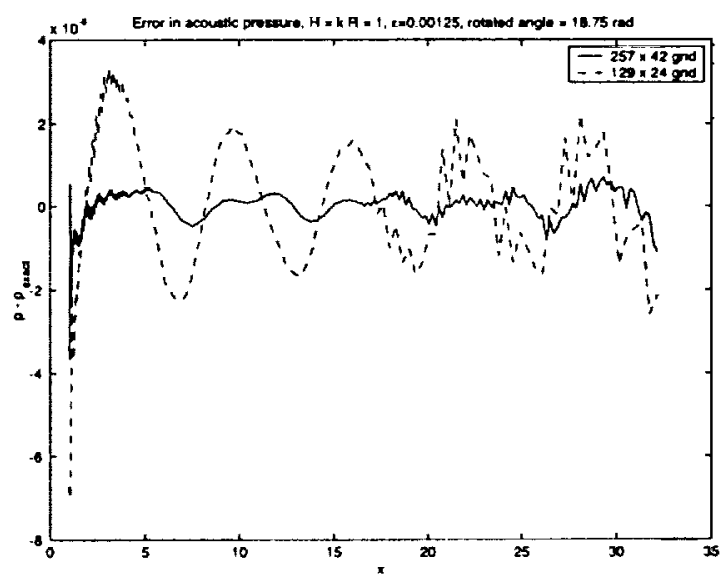

Fig. 10 Grid refinement study of error in acoustic pressure for instantaneously started generator with $\mathcal{H}=1, \epsilon=0.00125$, rotated angle $=18.75 \mathrm{rad}$.

to minimize the use of numerical dissipation where it is not needed (in this case, away from spurious oscillations). Thus, the results seem to be less sensitive to the filter coefficient $\kappa$, when the gradient detector switch is used.

The result of Yee et al. ${ }^{10}$ indicated that the use of entropy splitting of the inviscid flux derivative can minimize the use of numerical dissipation. For the present model problem, a similar conclusion as Yee et al. can be drawn. With entropy splitting, we can obtain reasonable results even without filter. Figures 13 and 14 compare the results without and with entropy splitting for $p^{\prime}$ along the positive $x$-axis. The improvement with entropy splitting is clearly visible. More results and the formulation of the entropy splitting for low Mach number aeroacoustics will be reported in Nüller and Yee 20 . 


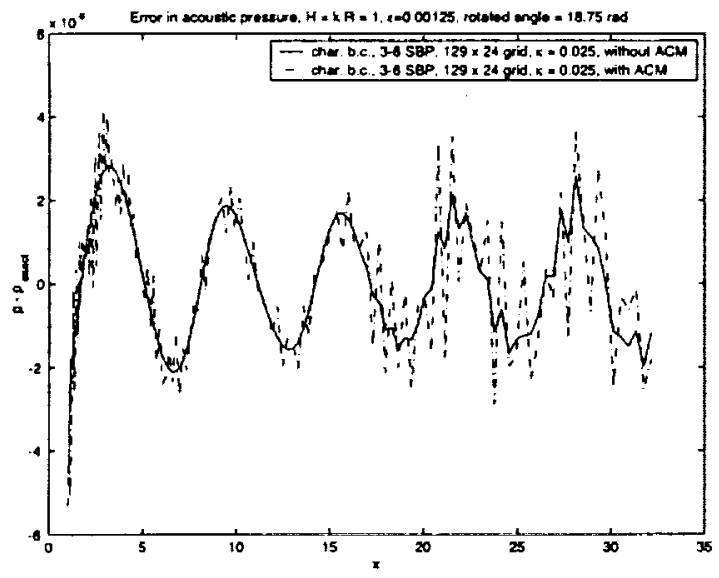

Fig. 11 Influence of the ACM sensor with $\kappa=0.025$ on error of acoustic pressure for instantaneously started generator with $\mathcal{H}=1, \epsilon=0.00125$, rotated angle $=18.75 \mathrm{rad}$.

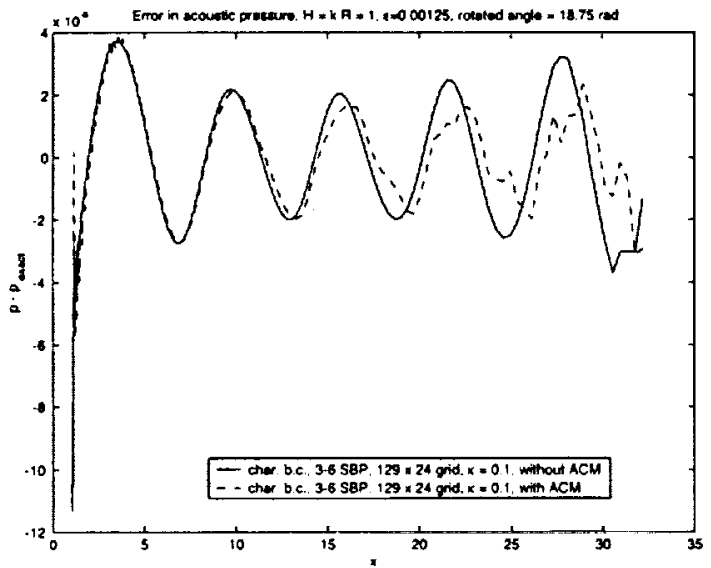

Fig. 12 Influence of the ACM sensor with $\kappa=0.1$ on error of acoustic pressure for instantaneously started generator with $\mathcal{H}=1, \epsilon=0.00125$, rotated angle $=18.75 \mathrm{rad}$

\section{Conclusions}

The periodic deformation of electrical generators by the rotors' magnetic fields is modelled as a rotating ellipse. The normal velocity of the elliptic generator surface provides the boundary condition for the acoustic field. For an almost circular generator surface, the Helmholtz equation governing the acoustic pressure is solved analytically in terms of the Hankel function of second order. Thus, the sound emission of rotor induced deformations of generator casings can be described analytically. The resulting model problem provides a good test case for the evaluation of CAA numerical algorithms.

Although the numerical simulation of the resulting almost circular Kirchhoff vortex model is similar to the study in Müller and Yee, ${ }^{16}$ a different form of the filter

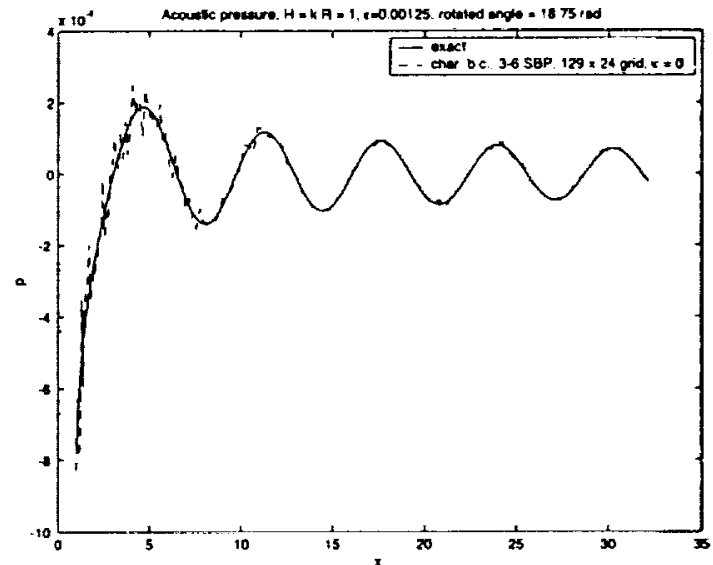

Fig. 13 Influence of no entropy splitting on acoustic pressure for instantaneously started generator with $\mathcal{H}=1, \epsilon=0.00125$, rotated angle $=18.75 \mathrm{rod}$.

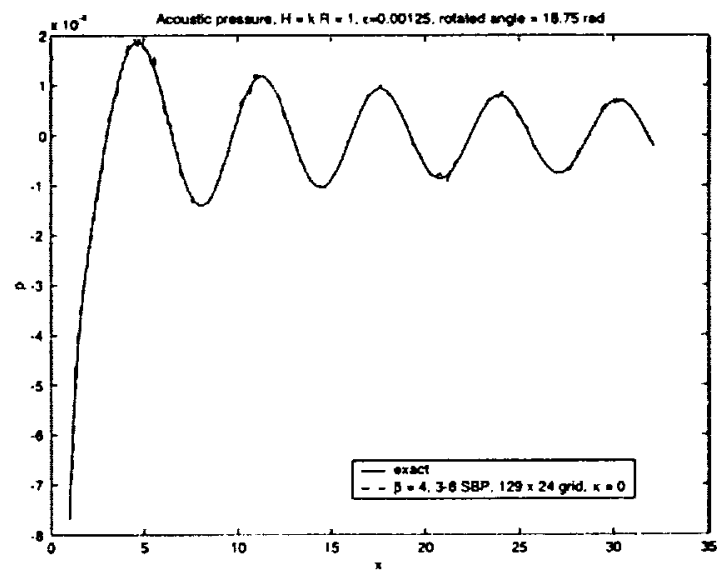

Fig. 14 Influence of entropy splitting on acoustic pressure for instantaneously started generator with $\mathcal{H}=1, \epsilon=0.00125$, rotated angle $=18.75 \mathrm{rad}$.

and temporal discretization are evaluated, and grid refinement study is also included. Results reveal a very accurate simulation with a fairly coarse grid for yet another important CAA physical application. For CAA flow problems of this type, especially for long time wave propagations, appropriate filter applied to the entropy splitting perturbation form of the Euler equations is essential to achieve stability and high accuracy of high order non-dissipative spatial base schemes.

\section{References}

${ }^{1} \mathrm{M}$. Ahrens, private communication, 1997.

${ }^{2}$ P.M. Morse and K.U. Ingard, Theoretical Acoustics, McGraw-Hill, 1968.

${ }^{3}$ B. Müller, "On Sound Generation by the Kirchhoff Vortex", Report No. 209/ 1998, Department of Scientific Computing, Uppsala University, October 1998. http://www.tdb.uu se/archive/reports/index.html

'Sesterhenn, J., Müller, B., and Thomann, H., "On the Cancellation Problem in Calculating Compressible Low Mach 
Number Flows," J. Comput. Physics, Vol. 151, 1999, pp. 597615.

${ }^{5}$ Kreiss, H.-O., Scherer, G., "Finite Element and Finite Difference Wethods for Hyperbolic Partial Differential Equations," Mathematical Aspects of Finite Elements in Partial Differential Equations. Academic Press, New York, 1974.

${ }^{6}$ Strand, B., "Summation by Parts for Finite Difference Approximations for $d / d x, " J$. Comput. Physics, Vol. 110, 1994, pp. 47-67.

${ }^{7}$ Carpenter, M.H., Gottlieb, D., Abarbanel, S., "TimeStable Boundary Conditions for Finite-Difference Schemes Solving Hyperbolic Systems: Methodology and Application to HighOrder Compact Schemes," J. Comput. Physics, Vol. 111, 1994, pp. 220-236.

${ }^{8}$ Gerritsen, M., Olsson, P., "Designing an Efficient Solution Strategy for Fluid Flows," J. Comput. Physics, Vol. 129 1996, pp. 245-262.

${ }^{5}$ Yee, H.C., Sandham, N.D., and Djomehri, M.J., "Low-Dissipative High-Order Shock-Capturing Methods Using Characteristic-Based Filters," J. Comput. Physics, Vol. 150, 1999, pp. 199-238.

${ }^{10}$ Yee, H.C., Vinokur, M., Djomehri, M.J., "Entropy Splitting and Numerical Dissipation," J. Comput. Physics, Vol. 162, 2000, pp. 33-81.

${ }^{11}$ Vinokur, M., Yee, H.C., "Extension of Efficient Low Dissipation High Order Schemes for 3-D Curvilinear Moving Grids," NASA TM 209598, 2000, and Proceedings of Computing the Future III: Frontiers in CFD - 2000, Half Moon Bay, CA, June 26-28, 2000.

${ }^{12}$ Olsson, P. "Summation by Parts, Projections and Stability. I," Math Comp., Vol. 64, No. 211, 1995, pp. 1035-1065.

${ }^{13}$ Olsson, P., "Summation by Parts, Projections and Stability. II," Moth Comp., Vol. 64, No. 212, 1995, pp. I473-1493.

${ }^{14}$ Olsson, P., Oliger, J., "Energy and Maximum Norm Es. timates for Nonlinear Conservation Laws," RIACS Technical Report 94.01, 1994.

${ }^{15}$ Olsson, P. "Surnmation by Parts, Projections and Stability. III," RIACS Technical Report 95.06, 1995.

${ }^{16}$ Müller, B., Yee, H.C., "High Order Numerical Simulation of Sound Generated by the Kirchhoff Vortex," RIACS Technical Report 01.02, Jan. 2001, NASA Ames Research Center.

${ }^{17}$ Carpenter, M.H., Gottlieb, D., Abarbanel, S., Don, W.S., "The Theoretical Accuracy of Runge-Kutta Time Discretization for the Initial Boundary Value Problem: A Study of the Boundary Error," SIAM J. Sci. Comput., 16, No. 6, 1241-1252 (1995).

${ }^{18}$ Fornberg, B., A Practical Guide to Pseudospectral Methods, First Paperhack Edition, Cambridge University Press, Cambridge, 1998.

${ }^{13}$ Johansson, M. "Loss of High Order Spatial Accuracy due to Boundary Error Caused by Runge-Kutta Time Integration," Technical Report 2000-013, Department of Information Technology, Uppsala University, May 2000. http://www.it.uu.se/research/reports/2000-013/

${ }^{20}$ Miüler, B., Yee, H.C. "Entropy Splitting for High Order Numerical Simulation of Vortex Sound at Low Mach Numbers," RIACS Technical Report 2001, NASA Ames Research Center, in preparation.

${ }^{21}$ Sjögreen, B., Yee, H.C., "Multiresolution Wavelet Based Adaptive Numerical Dissipation Control for Shock-Turbulence Computations," RIACS Technical Report 01.01, October 2000, NASA Ames Research Center. 